\title{
The potential use of drones to help natural pest control - an example using carabids
}

\author{
Gábor Lövei ${ }^{\ddagger}$, Annie Enkegaard§, Erik W Hansenl, Anders Petersen \\ $\ddagger$ Aarhus University, Aarhus, Denmark \\ $\S$ Department of Agroecology, Aarhus University, Flakkebjerg Research Centre, Slagelse, Denmark \\ | EWH BioProduction, DK-4733 Tappernøje, Denmark \\ I Ecobotix ApS, Slots Bjergby, DK-4200 Slagelse, Denmark
}

Corresponding author: Gábor Lövei (gabor.lovei@agro.au.dk)

Received: 05 Sep 2019 | Published: 05 Sep 2019

Citation: Lövei G, Enkegaard A, Hansen EW, Petersen A (2019) The potential use of drones to help natural pest control - an example using carabids. ARPHA Conference Abstracts 2: e46331. https://doi.org/10.3897/aca.2.e46331

\section{Abstract}

Small automated flying vehicles (drones, by common name) are undergoing very fast development, and the range of their potential use is also fast expanding. One possible application in agriculture is to disperse biological control agents in areas that are otherwise inaccessible, or the distribution of natural enemies is uneconomic for various reasons. We develop the use of multirotor type drones (DJI S-900) as a possible vehicle to fielddisperse carabid beetle adults as an inoculative biological control release against oilseed rape pests. We selected Pterostichus cupreus as a representative of common, larger species, and Anchomenus dorsalis as a similar representative of smaller species.

To test the robustness of these species to being dispersed by drones, we subjected the two carabid species to simulated "drop tests", using a specially modified 1 L-sized dispenser that can be fitted to the drone. We mixed 15-20 adults with two media, buckwheat bran and vermiculite and allowed the machine "dispersing" them under laboratory conditions. Subsequently, we checked the "distributed" beetles for mortality, bodily damage, and feeding capacity. Our first tests show that A.dorsalis is suitable for drone-based distribution while the larger species likely requires further modification of the dispensing system, due to the larger size and motility of this species. The dropping caused no mortality or physical damage to A.dorsalis but some of the P.cupreus lost tarsal segments. Feeding was not impaired in either species, though. 
In summary, drone technology opens promising possibilities for the widening of biological control possibilities in various situations in agriculture.

\section{Presenting author}

Gabor Lovei

Presented at

19thECM poster 